\section{Premature Infants}

This group contains all infants under $5 \frac{1}{2} \mathrm{lb}$. $(2.5 \mathrm{~kg}$.) birth weight, irrespective of the expected date of delivery. The systolic pressures that were recorded ranged from 35 to $70 \mathrm{~mm}$. $\mathrm{Hg}$, with a mean of 54 . The lower end of the range was recorded in two infants who survived only three days, and it should also be noted that one infant with a pressure of $50 \mathrm{~mm}$. $\mathrm{Hg}$ died on the first day. As shown in Figs. 2 and 3 the systolic pressure at birth was related to birth weight but not to maturity.

The premature infants attained values not significantly different from those of full-time infants by the third month, and the highest pressure recorded in any infant, $105 \mathrm{~mm}$. $\mathrm{Hg}$, at 6 months was in a prematurely born infant with a pressure of $50 \mathrm{~mm} . \mathrm{Hg}$ at birth.

\section{Twins}

There were four sets of twins in this series. Where one was anoxic it had the lower blood pressure; for instance, in one set of twins the first baby born did not breathe for five minutes and had a blood pressure $25 \mathrm{~mm}$. $\mathrm{Hg}$ below its sibling, who breathed spontaneously at once.

\section{Discussion}

The method of palpation which has been used to measure the mean systolic pressure of the newborn is simple and reliable and gives readily reproducible values suitable for comparison. Woodbury, Hamilton, and Robinow (1938) found that there was a close correlation in infants between the mean systolic pressure measured by an intra-arterial needle in the umbilical artery and by palpation of the brachial artery only when a $2.5-\mathrm{cm}$. cuff was used. The meihod also compares well with the flush method, recently assessed by Reinhold and Pym (1955), Sullivan and Kobayashi (1955), and Forfar and Kibel (1956), but is somewhat easier.

The systolic pressures in this series are higher than those stated by most previous investigators, but are similar to those found by Robinow et al. (1939), whose values ranged from 60 to $80 \mathrm{~mm}$. $\mathrm{Hg}$, and by Schaffer (1955), who used an impedance plethysmograph to record pulsation distal to an occlusion cuff $4 \mathrm{~cm}$. wide; his values for systolic pressure ranged from 52 to $102 \mathrm{~mm}$. $\mathrm{Hg}$, but nowhere does he state at what time after birth the measurements were made.

The low readings obtained before the simultaneous comparison of the cuff method with direct intra-arterial puncture by Woodbury et al. were probably due to the wide cuff used, for a width of at least $4 \mathrm{~cm}$. was the standard size for babies under 1 year of age (White House Conference, 1932). Balard (1912) using a Pachon oscillometer, found values of 55/35, and Reis and Chaloupka (1923), using a $6-\mathrm{cm}$. cuff and palpation, found values of $43 \mathrm{~mm}$. $\mathrm{Hg}$ (range $32-58$ ) on the first day, rising to $78 \mathrm{~mm}$. (52-94) by the tenth day.

The effect of the different conditions prevailing at birth upon the neonatal blood pressure was studied by Reis and Chaloupka and by Bowman (1933). The former included in their series three toxaemic pregnancies, and found that this condition had no effect on the systolic pressure of the newborn; this is in agreement with our own findings and those of Brasch (1949). Reis and Chaloupka also concluded that the more trauma to which the head was exposedthat is, the higher the forceps applied-and the larger the head, the higher would the blood pressure be. In two caesarean sections no effect on the blood pressure was noted, but there is no record at what interval after delivery these recordings were made. Bowman found in "cerebral" babies that the pressure was higher than normal, and was lowered by lumbar puncture. We were uncertain what was meant by this term, but if those cases emitting a "cerebral " cry are indicated, then in our series a lower systolic pressure than normal was found.

\section{Summary}

Palpation of the brachial artery below a $2.5-\mathrm{cm}$. inflatable cuff was used to measure the neonatal systolic blood pressure. The normal mean systolic pressure at birth was found to be $69 \mathrm{~mm}$. $\mathrm{Hg}$, rising to $93 \mathrm{~mm}$. $\mathrm{Hg}$ at 6 months. Foetal anoxia and abnormal forms of delivery such as caesarean section caused a significant lowering of systolic pressure at birth; the blood pressure of these babies recovered to normal levels within a few days. Drugs given to the mother before delivery and pre-eclamptic toxaemia had no effect on the neonatal systolic pressure at birth. The systolic pressure of premature babies was lower than that of full-term infants and was related to weight.

Our thanks are due to Dr. J. Forest Smith and Mr. A. J. Wrigley for allowing us to make these observations on their cases; to Sisters Gale and Harris and the midwives and nursing staff of Mary Ward for their kind help and co-operation; and to Professor Henry Barcroft for his encouragement and facilities.

REFERENCES

Balard, P. (1912). C.R. Soc. Biol. Paris, 73, 483

Bowman, J. E. (1933). Amer. J. Dis. Child., 46, 949.

Brasch, A. A. (1949). Arch. Dis. Childh., 24, 107.

Forfar, J. O., and Kibel, M. A. (1956). Ibid., 31, 126

Reis R., and Pym, M. (195s), I12., 30, 127.

Reis, R. A., and Chaloupka, A. J. (1923). Surg. Gynec. Obstet, 37, 206 , Hamilton, W. F . Woodbury, R. A., and Volpitto, P (1939). Amer. J. Dis. Child , 58, 102

Sullivan, M. P., and Kobayashi, M. (1955). Pediatrics, 15, 84

White House Conference (1932). Growth and Development of the Child, Part II, p. 294. Century Co. Growth Woodbury, R. A., Hamilton, W. F., and Robinow, M. (1938). Amer. J.
Physiol., 122, 472.

\section{CONGENITAL SPINAL PALSY}

BY

\section{F. ELLISON NASH, F.R.C.S.}

Surgeon, St. Bartholomew's Hospital, London; Sydenham Children's Hospital; and Heritage Hospital, Chailey, Sussex

With the reduction of infant mortality and of deaths from intercurrent infections in early childhood, many children are surviving with severe congenital deformities of a degree which has not hitherto formed a major clinical or social problem. The medical profession and the educational world are now fully aware of the needs of the cerebral palsy group, but surprisingly little attention has been paid to those afflicted with paraplegia in minor or major degree, from interference with the spinal cord. These for the most part are infants with spina bifida, usually but not always with a meningeal hernia. There are some with compression lesions of the cord due to severe deformity of the vertebral column and a few with permanent damage from birth injury or infective myelitis.

This paper is concerned primarily with the spina bifida palsies (Fig. 1). In the general run of surgical practice there are two distinct groups. The one is occult, said to be very common and alternately blamed and exonerated as a cause of wet beds in the lives of otherwise innocent children. Careful examination of some of these occult cases exposes a neurological abnormality. In some, micturition symptoms arise at or after adolescence owing to the development of uterus or prostate.

The second group is cystic. Where the cyst is a simple meningocele there is sometimes no cord or nerve 


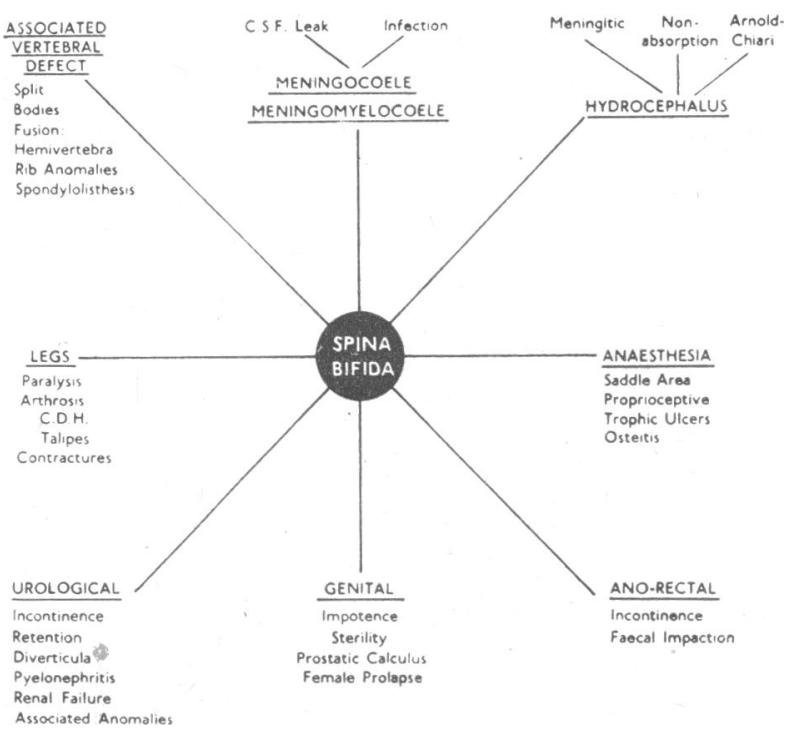

FIG. 1.-Complications of spina bifida.

involvement, though it is impossible to make such an assertion until the child has passed puberty and developed normal genito-urinary function. This second group is, however, largely that of the paraplegic incontinent cripple, who may be snatched from the jaws of death in the neonatal months, only to be delivered over to the tragedy of an expanding head or, in the past, to a life of social exclusion amid the fumes and filth of double incontinence and trophic ulcers. Deaths from this condition account for one-third of all infant deaths from congenital deformities.

\section{Incidence, Mortality, and Survival}

Over the last fifteen years for which statistics are available (1939-54) infant mortality in England and Wales has fallen from 50.57 to 25.42 per 1,000 live births. This represents a

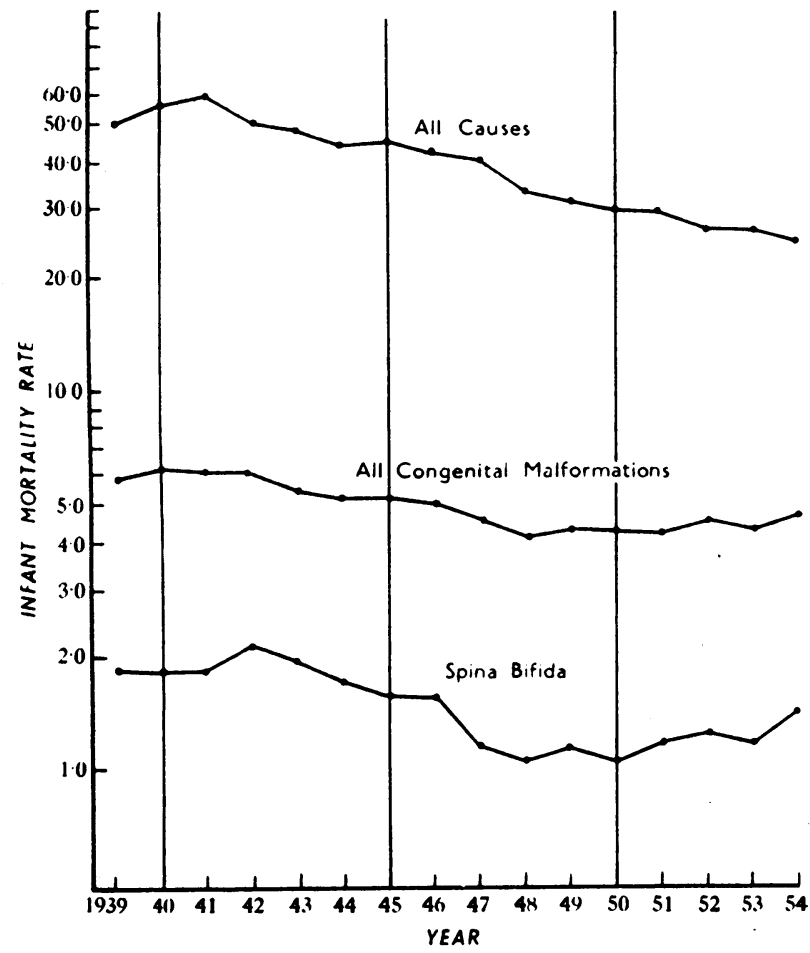

FIG. 2.-Infant mortality rates (per 1,000 live births) England and Wales, 1939-54. (Logarithmic scale.) reduction by one-half. The total number of deaths under the age of 1 year due to congenital malformations has dropped from 3,672 (5.93 per 1,000 live births) to 3,166 (4.69 per 1,000 live births) (Fig. 2). The infant deaths from spina bifida have fallen from 1,148 to 967 (Fig. 3). The

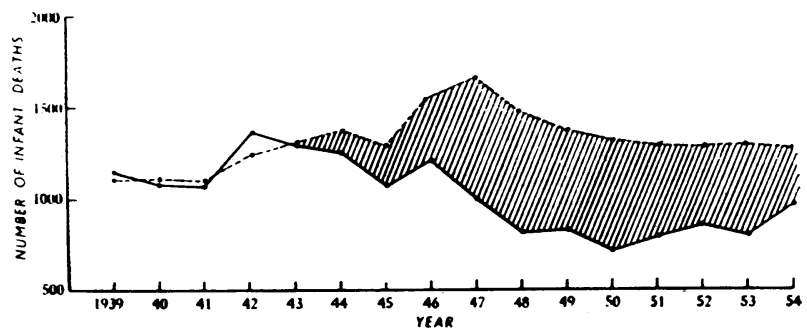

Fig. 3.- Infant deaths due to spina bifida (under 1 year) England and Wales, 1939-54. The broken line indicates the number of deaths from spina bifida which would have occurred if the infant mortality rate had remained at 1.9 per 1,000 live births-i.e., the average rate for 1940-4 (see Fig. 1). The shaded area therefore represents the probable salvage of severe cases which has occurred in the latter years.

lowest number (727) was in 1950. This striking reduction might at first seem due to postponement of death by antibiotics and improved care. It will be seen, however, that the deaths in the older age groups have not risen. It seems likely that an average of 450 to 500 severe cases are being salvaged each year, these representing the group of children who would have died before the antibiotic era.

A statistical survey of this and associated congenital defects in the central nervous system was reported by Record and McKeown (1949) from an exhaustive inquiry in the Birmingham area.

\section{Clinical Experience and Management}

The children who have formed the basis of my experience with this disability over the last ten years have come through three main channels. First, a small number have been referred in the neonatal period for an opinion on account of the meningocele. Second, there has been the ambulant group referred on account of urinary incontinence; many of these have come from orthopaedic colleagues in various centres. The third and most severe group is made up of those cases admitted to long-stay hospitals with which I am connected. A personal series of 65 survivors is under supervision. By and large, they represent the back-log of patients who would have been spared much of their disability if our present knowledge had been widely available ten years ago.

The management of these children is entirely different from that of the adult paraplegic whose disability has been acquired from disease or injury and who has a background of experience on which to build his recovery. The congenital paraplegic has never known leg movement, never "felt his feet," knows nothing of touch or pain below the groins, and has no pelvic visceral sensibility: there is no past experience on which to build new skills and adaptations. Ocular and vestibular mechanisms have not known the vertical position, and may have no spino-cerebellar pathways to co-ordinate their actions. Hitherto in the more severe cases of spinal palsy the subjects have passed through a period of devoted maternal care and have found themselves in the hands of orthopaedic surgeons when the normal walking age has been passed. Opportunities have been lost and secondary deformity is often grotesque. Early death from urinary infection or osteomyelitis has been common and the problem of school accommodation has not been a major one. Orthopaedic surgeons have been hesitant in the use of active corrective measures.

Suggestions concerning management of the various disabilities are put forward in the full recognition of the very wide range of disability that may occur. Each case requires careful analysis in respect of each deficiency. 


\section{The Primary Defect and its Treatment}

There is little certainty about the aetiology of this corrdition, but the primary defect is a herniation of the spinal meninges with or without cord or nerve-root involvement. The extent of the bony lesion is very variable. Aetiology and pathology of this and associated primary defects in the central nervous system are fully described by Morison (1952) and Cameron (1956).

Hydrocephalus is commonly associated with spina bifida, and the two conditions may have a common origin in the failure of development of the cerebrospinal fluid absorption mechanism, the spinal anomaly then arising as a "blow-out." The canal closes cranio-caudally so that the most common site for the defect would on this hypothesis be in the lumbosacral area, as is indeed the case. Spina bifida occulta, with no backward protrusion, may also be associated with nerve defect; sometimes the site of neural arch involvement is marked by a port-wine stain on the skin, or by a tuft of hair.

A considerable number of fatal cases are found to have Arnold-Chiari deformity. In these cervical and thoracic regions minor meningoceles occur, but there is only very occasional nerve involvement in these. In the lumbar and lumbosacral region, where the defect is most common, even a small gap may be associated with quite severe involvement of the spinal nerves. If the defect extends above the level of the second lumbar vertebra there is usually a broadbased meningomyelocele, and the condition is more often fatal. Meningitis is a rare complication except in those with extensive herniation. If a baby survives this ascending infection, hydrocephalus may ensue from basal meningeal adhesions. Operative intervention to prevent infection in the more gross cases with a cerebrospinal leak rarely prevents death. The survivors therefore are mostly those with low lesions and cauda equina involvement.

Early operative removal is justified only if the meningocele sac has a narrow pedicle. Even the tumours with a moist centre of neural tissue will become covered by skin growing in from the edges. Those with a very thin transparent epithelium come to no harm if they are protected; the covering skin thickens and often the tumour shrinks in the first few months of life, so that no subsequent treatment is needed. Small cerebrospinal fistulae close spontanously, but a major rupture demands immediate surgery. Some of the healed thickened plaques remain very sensitive, and excision may be needed, but this should be postponed until maximum functional development of sphincters and limbs has been achieved.

With these exceptions, operation upon the tumour is avoided. It may precipitate the onset of hydrocephalus or aggravate that condition if it is already present. Sterile non-adherent dressings are held in place by a padded ring made to fit the tumour and prevent pressure. The presence of this lump in the lumbar region prevents the child sleeping on its back, and because of this continued lateral posture the natural inclination is for the hips and knees to become fixed in flexion. Protection later on can be provided by half a child's rubber ball, perforated at several points to allow ventilation. A large coffee-strainer with the handle removed is a more rigid guard, and can be fixed in position over a disk of adhesive felt. Very exceptionally a large and cumbersome tumour may require excision ultimately and a cerebral posterior decompression or coelomic drainage may be needed to prevent hydrocephalus (see below).

\section{Associated Hydrocephalus}

Spina bifida cystica may occur in association with hydrocephalus as a double primary anomaly. Such cases usually exhibit cranial enlargement at birth, and this is rapidly progressive. Hydrocephalus may arise during the first year from a mild arachnoiditis which either tethers the cord or interferes with the normal cerebrospinal fluid circulation at the base of the brain. Macnab (1954) recorded that 23 out of 27 babies with spina bifida cystica and progressive hydrocephalus gave evidence of the progression by the fourth month of life.

In many cases the hydrocephalus seems to be arrested spontaneously, so that intervention can be justified only in the presence of rapidly increasing head measurement. Operative relief of the intracranial pressure has been attempted in various ways (Jolly, 1955; Macnab, 1955). The most satisfactory method appears to be the formation of a fistula between the theca and the peritoneum by means of a "polythene" tube.

Radiographic air studies may be advisable to assess the extent of intracranial damage, but as the presence of a minor degree of hydrocephalus does not affect the treatment of the child the risk of investigation is not justified as routine. Hydrocephalus seems to bear little relation to intelligence or educability.

\section{Motor Defect}

A child's motor ability is affected in three ways. First, by direct interruption of the motor axons by spinal cord or cauda equina damage. This represents the permanent and irretrievable loss. Macnab and Cameron (Macnab, 1954) showed from examination of the post-mortem material that myelinization of the distorted cord was delayed or incomplete : this would explain the relatively late appearance of movements in limbs which have been previously found completely flaccid. This possibility of late development is a further reason against early operative intervention for removal of the spinal tumour. There is little doubt that many children have been operated on prematurely and have thus been deprived of useful leg movement.

Second, there may be, as an associated primary defect, congenital dislocation of one or both hips. Pathological dislocation may occur subsequent to pelvic muscle paralysis. In the past the hip lesions have been regarded too lightly by orthopaedic surgeons, and the untreated dislocation has contributed materially to the overall disability. Every effort should be made to maintain leg length and prevent persistent dislocation.

Third, paralysis and dislocation if untreated lead to the development of severe muscle contractures, particularly of psoas, rectus femoris, and hamstrings. Dislocation of the hips may actually result from adductor and psoas contracture. Foot deformities should be manipulated and splinted, great care being taken to avoid pressure sores. Though much can be attained by late correction. these deformities should never be allowed to occur.

In older children where neglected deformity makes balance and ambulation impossible, excision of joints may be necessary to produce a flail limb during the growth period. Arthrodesis of the foot at the appropriate age may also be called for. A defeatist attitude must not be allowed to develop. Urinary incontinence may interfere with ambulation and with orthopaedic appliances, and it must be dealt with appropriately (see below).

\section{Sensory Defect}

The distribution of cutaneous and deep anaesthesia is very variable. There may be no detectable sensory loss on clinical examination but the rectum and urethra may nevertheless be anaesthetic, with subsequent constipation and retention of urine. "Saddle" anaesthesia leads to the development of deep sores over weight-bearing points at the ischial tuberosities or great trochanters. The heels and malleoli very easily become blistered and penetrating ulcers under metatarsal heads lead to infective osteitis of the metatarsal bones. Long-term antibiotic therapy must be used if bone infection is present, and penicillin may be continued for many months if the organism is sensitive (Nash, 1951). Lumbar sympathectomy has an established place in the management of children who develop severe chilblains, and a really material improvement in circulation may be obtained so that trophic ulcers heal. Where there 
is doubt about the nutrition of the skin and an orthopaedic operation on the limbs is contemplated, lumbar sympathectomy is a wise preliminary measure.

If motor power above the knees is satisfactory, a deformed and ulcerated foot may be best dealt with by amputation.

Boots and shoes should always have lamb's-skin linings.

On the buttocks, thighs, and perineum, ulcers develop urinary dermatitis. In the early years of life, when the urine is relatively dilute, the normal napkin regime is adequate With a larger child, waterproof knickers ought to be avoided, but unless the incontinence is adequately dealt with it is impossible to prevent the development of sodden ulcers on the external genital organs. Ulceration over the ischial tuberosity leads to necrosis of the underlying fat, which has to be excised or be extruded as a slough.

Gentian-violet paint is a suitable hardening antiseptic for sores in the saddle area. Silicone barrier creams are of great value in preventing dermatitis and eliminating "chapping" of the skin on the adductor areas of the thigh. The liberal use of silicone creams on the pressure points of bedridden patients greatly diminishes the risk of trophic ulceration (Bateman, 1956).

\section{Urinary Tract}

With spina bifida there is a frequent association of congenital abnormality in the urinary tract. The one may affect the other

Reduplications, misplacement, malrotation, fusion (the horseshoe kidney), or agenesis of one side may be found. The presenting symptoms may be due to infection superimposed upon such primary anatomical defects. Neverthelesss, the urinary symptoms, and indeed the infection, are usually due to the neurogenic disturbance of the micturition mechanism.

In cord and cauda equina lesions the alterations in bladder mechanism are both sensory and motor. With spina bifida there is no clear-cut transection of the spinal cord, nor is there any segmental correspondence between the sensory and the visceromotor loss. If the urethral sensation is defective or the sensation of bladder-wall stretch is absent then there will be neither the impulse to start micturition nor the ability to control a regular flow. If the detrusor muscle lacks adequate innervation, or if there is imbalance between the main detrusor and that part of it which forms the bladder-neck muscle, there is retention. This retention can occur in the presence of a grossly hypertrophic bladder or with one in which the detrusor is thin and multiple diverticula are present.

Paralysis of the external sphincter leads to complete incontinence if there is no obstruction of the bladder neck. Varying degrees of sphincter dysfunction are found, and the net result of any of these is that the child is incontinent, whether with dribbling micturition or with retention overflow.

It is difficult to assess the degree of involvement of either vesical or rectal innervation in the infant. The most vital requirement is that the bladder should empty adequately. In the first few years of life, when urinary incontinence is acceptable, the clinician's duty is to treat and prevent infection, having made quite certain that there is no significant residual urine-that is, not more than $2 \mathrm{oz}$. $(57 \mathrm{ml}$.) at the age of 5. Fortunately, the automatic function of the infant bladder seems to be preserved even in those most severely affected; but after the first year many of the children develop progressive retention. The subsequent back-pressure effect on the upper tract is the complication which has proved to be fatal so often in the past.

So far as survival is concerned, therefore, the care of the urinary tract is of paramount importance. It is necessary in every case to undertake a complete investigation of the urinary tract by excretion pyelogram and by instrumentation. The measurement of the residual urine must be performed at regular intervals and the blood urea estimated at the same time. Tests for renal function, apart from the assessment of the specific gravity of the urine, are apt to be misleading, and the urea-clearance test is useless if there is significant dilatation of any part of the tract or any residual urine.

After investigation it is found that the cases fall clearly into one of several groups : (1) complete incontinence, without residual urine ; $(2)$ complete or intermittent incontinence, with a small volume of residual urine; this may be managed satisfactorily by manual expression and antiinfective measures; and (3) retention with overflow incontinence-usually there is distinctive back-pressure change in the urinary tract.

\section{Surgical Measures}

Resection of the bladder neck or suprapubic catheter drainage is necessary for the relief of obstruction. Resection in girls often leads to vesico-vaginal fistula and total incontinence. Suprapubic drainage is never very satisfactory, as infection is always severe and leakage common. Pudendal neurectomy is sometimes an alternative or additional procedure to resection of the bladder neck. The control of incontinence-primary in group 1 , or following surgical intervention in group 3 cases--is now possible in every case. Penile urinals are sometimes satisfactory but always hazardous. Special designs are available to lessen the risk of ulceration. Urethral plication in the male-an operation orginally designed for post-prostatectomy incontinenze-has been used with complete success in several cases.

\section{Ileal Bladder}

The use of an isolated ileal loop to form an ileo-cutaneous ureterostomy has completely changed the outlook for the incontinent girls, and for the boys when other measures have failed.

Valuable contributions to the problem of incontinence have been made by those who have pioneered the use of an isolated ileal segment as a bladder (Annis et al., 1954). Bill et al. (1954) and Pyrah (1956) have described the use of this operation in cases of spina bifida.

The two ureters are detached from the bladder and implanted into the pelvic end of a loop of ileum which hangs on its mesenteric segment below the normal ileal mesentery. Continuity of the bowel is restored by end-to-end anastomosis and the distal end of the isolated urine-bearing segment is delivered through the abdominal wall in the right iliac fossa. Its end is everted to form a mucosa-covered spout, over which is mounted, by an adhesive patch, a light flanged latex ileostomy (Rutzen) bag. A tap is fitted to the bottom of the bag.

I have now used this technique in 10 cases. The improvement in the general health and well-being of all these young patients has been dramatic and most gratifying. Urinary infection is practically eliminated. The operation carries considerable immediate post-operative risk, but the later hazards are much less than those of petsistent incontinence with or without back-pressure. The result is complete freedom from urine contamination. The genital ulcers heal and ambulation is greatly simplified. Three of these children have been able to return to ordinary day schools, travelling in buses and caring for themselves. One girl died six months after operation. She had long-standing severe pyelonephritis and an Arnold-Chiari brain defect. The others, some with severe orthopaedic deformities, are still in hospital schools or attending special schools from their homes; two adolescents are at work. They are well, and are very pleased with their new freedom.

I consider that the introduction of this operation has revolutionized the prognosis for the unfortunate victims of this condition. Spinal palsy has taken a new turn towards becoming a disease in which there is a reasonable possibility of good education, adaptation, social acceptance, and employment.

\section{Bowel Control}

The initiation of rectal contraction in the infant is entirely reflex. Later, under voluntary control it is stimulated by the rise of abdominal pressure produced by straining. In 
spinal palsy there is impairment of the sensory side of the reflex arc and constipation is the rule.

Unless the stool is soft or fluid, incontinence does not occur even in the presence of sphincter impairment. On clinical examination of cases of spina bifida, the anus is found to be patulous in almost all cases. Nevertheless a certain degree of voluntary control exists in some, and this seems to depend largely on the acquisition of habit and the ability to defaecate by abdominal straining. Any gastrointestinal disturbance such as occurs with infective diarrhoea or dietetic upset leads to incontinence: such a disturbance usually produces colic, and the patient is therefore aware of what is likely to happen, although once the irritation has reached the rectum there can be no further warning; uncontrolled defaecation is then inevitable.

The principal difficulty in the management of these children is that they suffer from faecal impaction. As the result of this they may in fact be incontinent, but it is an overflow incontinence and can be entirely eliminated by regular enemas until habit evacuation is restored. The use of " aerosol OT" by mouth ( $2 \%, 4 \mathrm{ml}$., increasing as required) or as a retention enema helps in the dehiscence of these faecal masses. If the impaction is troublesome the use of small quantities of paraffin emulsion is justified; but, even in moderate dosage, leakage may occur, and under no circumstances should non-emulsified paraffin be used.

No case in my series has failed to respond to this training. Faecal incontinence should therefore not be accepted: its occurrence-except as a rare incident-is a reflection on those responsible for the management of the child. Weakness of levator ani might be expected to result in rectal prolapse, but I have never seen this. The absence of this complication may be due to the fact that sensation is impaired and therefore there is no straining. Colostomy is never indicated and can only add to the misery. In the older children and adults, if a particular social occasion is causing anxiety from fear of faecal incontinence (particularly in those individuals whose bowel responds to emotional stress), the judicious use of codeine compound tablets will give adequate constipation and security. Glycerin suppositories are an adequate stimulus to evacuation of the bowel in some children, and I have found that over a period of months the mother discovers what is most suitable to achieve regular action and reliability. There is no fear of producing abnormal psychological states by treatment, as the sensory loss is such that erotic stimuli cannot occur.

\section{Education}

The educational deficit begins to build up in the toddler period, since normal childhood experiences are denied to those who are unable to walk and mix with other children. There is a natural tendency to over-protection on the mother's part which always accompanies such disability; many of these children are extremely sensitive and lack the fibre of physically normal children of the same age group. When the child might normally be attending a day nursery, or even primary school, accommodation is not always available, or the mother may not be willing to allow the child to travel in the school bus. Repeated ulceration, urinary infection, and other complications result in an intermittent school attendance even in those for whom accommodation and adequate provision are made. Similarly, in residential schools, unless there is scrupulous care to prevent the occurrence of trophic ulceration, the same set of disabilities arises and results in loss of school time. The necessarily more boisterous and less protected life of schoolgoing children may prove too tough.

Hard chairs and lack of really individual supervision make life in an ordinary general school for physically handicapped children particularly hazardous for the incontinent paraplegic. There is a good case to be made out for schools specially set apart and equipped for this type of child. While the aim must always be that a disabled child is able to live in its own home and attend a day school if social conditions permit, it is quite clear that with these severe and complicated disablements it is often very much better for the child to be in a special residential or hospital school. Here the advantages of clinical improvement and continuous education far outweigh the disadvantages of the child missing its normal home life. It is of course essential that units dealing with this as with other types of disabled children should be small enough for there to be something of a family spirit to replace what the child misses by absence from its own home. Maladjustment is a rarity, probably because of the naturally sheltered existence which these children lead.

\section{Discussion}

The changing pattern of child disablement in the last two decades has resulted in a tremendous reduction in the number of children with tuberculous or pyogenic bone and joint infection. Disability from poliomyelitis is still with us, but is probably now of transient interest so far as future hospital and school provision is concerned.

With the reduced mortality in the past ten years we can assume the survival of large numbers of infants severely affected with congenital spinal palsy. The slight increase of infant deaths from spina bifida in the last three years (some 200 more deaths) may be due to more successful obstetrics and the initial survival of babies who might previously have been stillborn.

This special group of disabled children is now accumulating in our special schools and many cases are still being cared for in their own homes. It is impossible to assess accurately what additional hospital and school accommodation may be necessary. There is little doubt that the accommodation for the adolescent paraplegic with a very severe handicap is inadequate.

\section{Summary}

The significance of congenital defects of the spinal cord is emphasized as a growing social problem. The implications in relation to the varied disability pattern are outlined, particularly in relation to urinary and faecal incontinence. Practical measures are suggested upon which a programme of clinical and educational management should be built for each child. Surgical procedures include lumbar sympathectomy, urethral plication, resection of the bladder neck, and ileocutaneous ureterostomy. Particular emphasis is placed upon the necessity for early and continuous supervision to prevent sequelae which have in the past eliminated these patients from commercial life and useful employment.

No attempt has been made to deal in detail with any one aspect of this complex problem but to focus attention on the need to integrate all our resources in the interests of this group and to emphasize the major contribution which can be made by both urological and general surgery. Corrective orthopaedic measures which are so ably applied in many cases have in the past been withheld from others because of the apparent hopelessness of the child's condition as a whole. This despondent attitude on account of urinary and faecal incontinence is no longer justified.

I must pay tribute to the devotion and care which has been shown by nursing staffs concerned in the management of my patients in this group, and I acknowledge in particular the help and interest of Dr. P. Quibell.

\section{REFERENCES}

Annis, D., Hunter, W. R., and Wells, C. (1954). Brit. J. Surg., 42, 290. Bateman, F. J. A. (1956). British Medical Journal, 1, 554.

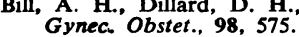

Cameron, A. H. (1956). Lancet, 2, 171.

Jolly, H. (1955). Proc. roy. Soc. Med., 48, 843.

Macnab, G. H. (1954). Ann. roy. Coll. Surg. Engl., 14, 124.

- (1955). Proc. roy. Soc. Med., 48, 846.

Morison, J. E. (1952). Foetal and Neonatal Pathology. Butterworth,

London.
Nash, D. F. Ellison (1951). Surgical Progress. Butterworth, London.

Pash, D. F. Ellison (1951). Surgical Progress. Butter

Record $\dot{\mathbf{R}}$ G. 